\title{
COFACTORS AND EIGENVECTORS OF BANDED TOEPLITZ MATRICES: TRENCH FORMULAS VIA SKEW SCHUR POLYNOMIALS
}

\author{
Egor A. Maximenko and Mario Alberto Moctezuma-Salazar
}

Abstract. The Jacobi-Trudi formulas imply that the minors of the banded Toeplitz matrices can be written as certain skew Schur polynomials. In 2012, Alexandersson expressed the corresponding skew partitions in terms of the indices of the struck-out rows and columns. In the present paper, we develop the same idea and obtain some new applications. First, we prove a slight generalization and modification of Alexandersson's formula. Then, we deduce corollaries about the cofactors and eigenvectors of banded Toeplitz matrices, and give new simple proofs to the corresponding formulas published by Trench in 1985.

Mathematics subject classification (2010): 05E05, 15B05, 11C20, 15A09, 15A18. Keywords and phrases: Toeplitz matrix, skew Schur function, minor, cofactor, eigenvector.

\section{REFERENCES}

[1] P. AleXAndersson, Schur polynomials, banded Toeplitz matrices and Widom's formula, Electron. J. Combin. 19, 4 (2012), P22, http://www.combinatorics.org/ ojs/index.php/eljc/article/view/v19i4p22.

[2] M. BARRERA AND S. M. GRUDSKY, Asymptotics of eigenvalues for pentadiagonal symmetric Toeplitz matrices, Oper. Theory Adv. Appl. 259 (2017), 51-77, doi: 10.1007/978-3-319-49182-0_7.

[3] G. BAXTER AND P. SCHMIDT, Determinants of a certain class of non-Hermitian Toeplitz matrices, Math. Scand. 9 (1961), 122-128, http://www.mscand.dk/article/view/10630/8651.

[4] J. M. Bogoya AND A. BötTCher AND S. M. GRudSKY AND E. A. MAKSIMENKo, Eigenvectors of Hessenberg Toeplitz matrices and a problem by Dai, Geary, and Kadanoff, Linear Algebra Appl. 436 (2012), 3480-3492, doi: 10.1016/j.laa.2011.12.012.

[5] J. M. Bogoya And A. Böttcher and S. M. Grudsky And E. A. Maximenko, Maximum norm versions of the Szegó and Avram-Parter theorems for Toeplitz matrices, J. Approx. Theory 196 (2015), 79-100, doi: 10.1016/j.jat.2015.03.003.

[6] J. M. Bogoya And A. Böttcher And S. M. Grudsky and E. A. Maximenko, Eigenvectors of Hermitian Toeplitz matrices with smooth simple-loop symbols, Linear Algebra Appl. 493 (2016), 606-637, doi: 10.1016/j.laa.2015.12.017.

[7] A. BORODIN AND A. OKOUNKOV, A Fredholm determinant formula for Toeplitz determinants, Integr. Equ. Oper. Theory 37, 4 (2000), 386-396, doi: 10.1007/BF01192827.

[8] A. Böttcher And S. M. GRudsky, Spectral Properties of Banded Toeplitz Matrices, SIAM, Philadelphia, 2005, doi: 10.1137/1.9780898717853.

[9] A. Böttcher And S. M. GRudsky And E. A. Maksimenko, On the structure of the eigenvectors of large Hermitian Toeplitz band matrices, Oper. Theory Adv. Appl. 210 (2010), 15-36, doi: 10.1007/978-3-0346-0548-9_2.

[10] A. Böttcher and L. Fukshansky and S. R. Garcia and H. Maharaj, Toeplitz determinants with perturbations in the corners, J. Funct. Analysis 268, 1 (2015), 171-193, doi: $10.1016 / \mathrm{j} \cdot \mathrm{jfa} \cdot 2014 \cdot 10.023$.

[11] A. Böttcher And B. Silbermann, Introduction to Large Truncated Toeplitz Matrices, SpringerVerlag, New York, 1999. 
[12] A. Böttcher and B. Silbermann, Analysis of Toeplitz Operators, 2nd ed., Springer-Verlag, Berlin, Heidelberg, New York, 2006, doi: 10.1007/3-540-32436-4.

[13] A. Böttcher And H. Widom, Szegö via Jacobi, Linear Algebra Appl. 419 (2006), 656-667, doi: 10.1016/j. laa.2006.06.009.

[14] D. Bump And P. Diaconis, Toeplitz minors, J. Combin. Theory Ser. A 97 (2002), 252-271, doi: 10.1006/jcta.2001.3214.

[15] P. Deift And A. ITS And I. Krasovsky, Toeplitz matrices and Toeplitz determinants under the impetus of the Ising model. Some history and some recent results, Comm. Pure Appl. Math. 66, 9 (2013), 1360-1438, doi: 10.1002/cpa.21467.

[16] M. ElouafI, On a relationship between Chebyshev polynomials and Toeplitz determinants, Appl. Math. Comput. 229, 1 (2015), 27-33, doi: 10.1016/j . amc.2013.12.029.

[17] M. Elouafi, A Widom like formula for some Toeplitz plus Hankel determinants, J. Math. Anal. Appl. 422, 1 (2015), 240-249, doi: 10.1016/j.jmaa.2014.08.043.

[18] C. GARONi AND S. SerRa-CAPIZZAno, The theory of locally Toeplitz sequences: a review, an extension, and a few representative applications, Bol. Soc. Mat. Mex. 22, 2 (2016), 529-565, doi: $10.1007 / \mathrm{s} 40590-016-0088-8$.

[19] I. M. Gessel, Symmetric functions and P-recursiveness, J. Combin. Theory, Ser. A 53, 2 (1990), 257-285, doi: 10.1016/0097-3165(90)90060-A.

[20] U. Grenander And G. Szegő, Toeplitz Forms and Their Applications, University of California Press, Berkeley, Los Angeles, 1958.

[21] A. LASCOUX, Symmetric Functions and Combinatorial Operators on Polynomials, In series: CBMS Regional Conference Series in Mathematics, vol. 99, co-publication of the AMS and Conference Board of the Mathematical Sciences Providence, Rhode Island, 2003, doi: 10.1090/cbms/099.

[22] D. E. LitTlewood, The Theory of Group Characters and Matrix Representations of Groups, 2nd ed. The Clarendon Press, Oxford University Press, Oxford, 1950.

[23] I. G. Macdonald, Symmetric functions and Hall polynomials, 2nd ed. The Clarendon Press, Oxford University Press, Oxford, 1995.

[24] V. Reiner And K. M. ShaW And S. VAn Willigenburg, Coincidences among skew Schur functions, Adv. Math. 216, 1 (2007), 118-152, doi: 10.1016/j . aim.2007.05.006.

[25] The Sage Developers, SageMath, the Sage Mathematics Software System (Version 7.5.1) (2017), doi: $10.5281 /$ zenodo. 28514, http://www. sagemath.org.

[26] The Sage-Combinat community, Sage-Combinat: enhancing Sage as a toolbox for computer exploration in algebraic combinatorics, (2008), http://combinat. sagemath.org.

[27] R. P. Stanley, Enumerative Combinatorics, vol. 2. Cambridge University Press, Cambridge, 1999.

[28] C. A. TRACY, H. WidOM, On the distributions of the lengths of the longest monotone subsequences in random words, Probab. Theory Relat. Fields 119 (2001), 350-380, doi: 10.1007/s004400000107.

[29] W. F. TRENCH, Explicit inversion formulas for Toeplitz band matrices, SIAM J. on Algebraic and Discrete Methods 6, 4 (1985), 546-554, doi: 10.1137/0606054.

[30] W. F. Trench, On the eigenvalue problem for Toeplitz band matrices, Linear Algebra Appl. 64 (1985), 199-214, doi: 10.1016/0024-3795 (85) 90277-0.

[31] H. Widom, On the eigenvalues of certain Hermitean operators, Trans. Amer. Math. Soc. 88, 2 (1958), 491-522, doi: $10.2307 / 1993228$. 